\title{
Langerhans Cell Histiocytosis of the Temporal Bone in Children: Two Cases Report
}

\author{
Y Labani, O Oulghoul, O Benhoummad, Y Rochdi, and A Raji
}

\section{ABSTRACT}

Langerhans cell histiocytosis is a rare hematologic disease due to multiplication and accumulation of Langerhans cells, which are immature dendritic cells. The clinical presentation is highly variable and can range from isolated, self-healing skin or bone lesions to life-threatening multisystem disease. We report 2 cases confirmed by Histological and immunohistochemical evaluation of temporal bone biopsies. The first case is a 2-year-old child with chronic bilateral otorrhea and polyuria-polydipsia syndrome. Physical examination showed bilateral retroauricular redness and induration with filling all of the right and left external ear canal. Computed tomography showed osseous lysis of the temporal bone bilaterally. Magnetic resonance imaging of the brain showed the presence of pituitary infiltration. The second case is 2-year-old girl with chronic left otorrhea. Clinical examination objectified the presence of redness and induration in the left mastoid with the presence of an inflammatory polyp filling the entire left external auditory canal. Computed tomography revealed a left temporal bone lysis. Both patients were treated with chemotherapy.

Keywords: Children, Langerhans cell histiocytosis, Temporal bone.

Submitted : April 28, 2021

Published : May 24, 2021

ISSN: $2593-8339$

DOI: $10.24018 /$ ejmed.2021.3.3.839

Y. Labani *

ENT-HNS Department, Mohammed VI

University Hospital, Morocco.

(e-mail: youness.labani ${ }^{\circledR}$ gmail.com)

O. Oulghoul

ENT-HNS Department, Mohammed VI

University Hospital, Morocco.

O. Benhoummad

ENT-HNS Department, Mohammed VI

University Hospital, Morocco.

Y. Rochdi

ENT-HNS Department, Mohammed VI

University Hospital, Morocco.

A. Raji

ENT-HNS Department, Mohammed VI

University Hospital, Morocco.

*Corresponding Author

\section{INTRODUCTION}

Langerhans cell histiocytosis $(\mathrm{LCH})$ is a rare hematologic disease due to multiplication and accumulation of Langerhans cells, which are immature dendritic cells [1]. The clinical presentation is highly variable and can range from isolated, self-healing skin or bone lesions to life-threatening multisystem disease. We report two cases: a 2-year-old child with bilateral temporal and pituitary involvement and a 2year-old girl with unilateral left temporal involvement.

\section{Clinical CASe 1}

A 2-year-old child, with no particular medical history, presented for 6 months a polyuropolydipsic syndrome with chronic bilateral otorrhea. The child was conscious, hemodynamically and respiratory stable, and with no signs of dehydration. Clinical examination revealed the presence of bilateral retro auricular redness and induration. Otoscopy revealed the presence of inflammatory polyps filling both of the right and left external auditory canals. Blood work was done glucose was normal at $0.8 \mathrm{~g} / \mathrm{L}$, urinary osmolality was decreased to $112 \mathrm{mOsm} / \mathrm{L}$ and blood serum was normal at $140 \mathrm{mmol} / \mathrm{L}$. Urinary osmolality was low even after fluid restriction test. Urinary osmolality increased to $326 \mathrm{mOsm} / \mathrm{L}$ after 4 hours of administration of $10 \mu \mathrm{g}$ of Desmopressin (Minirin ${ }^{\circledR}$ ) spray. The diagnosis of central diabetes insipidus was retained. Brain magnetic resonance imaging (MRI) showed the presence of pituitary infiltration with enlargement of the pituitary stem enhancing to $\mathrm{T} 1$ sequences after injection of contrast agent (Fig. 1). Computed tomography (CT) revealed bone lysis involving the temporal bone bilaterally (Fig. 2). A deep biopsy is done under general anesthesia by a retro auricular approach. Histological study objectified the presence of a fibrous tissue seat of a cellular infiltrate made up of inflammatory cells and histiocytic cells (Fig. 3), Immuno-markers were positive for PS100, CD1a and CD68 confirming the diagnostic of $\mathrm{LCH}$.

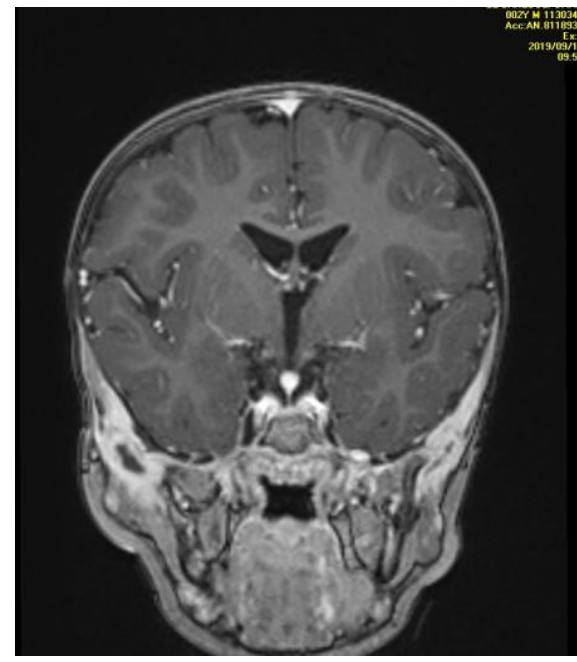

Fig. 1. Cerebral MRI in coronal section showing pituitary infiltration. 


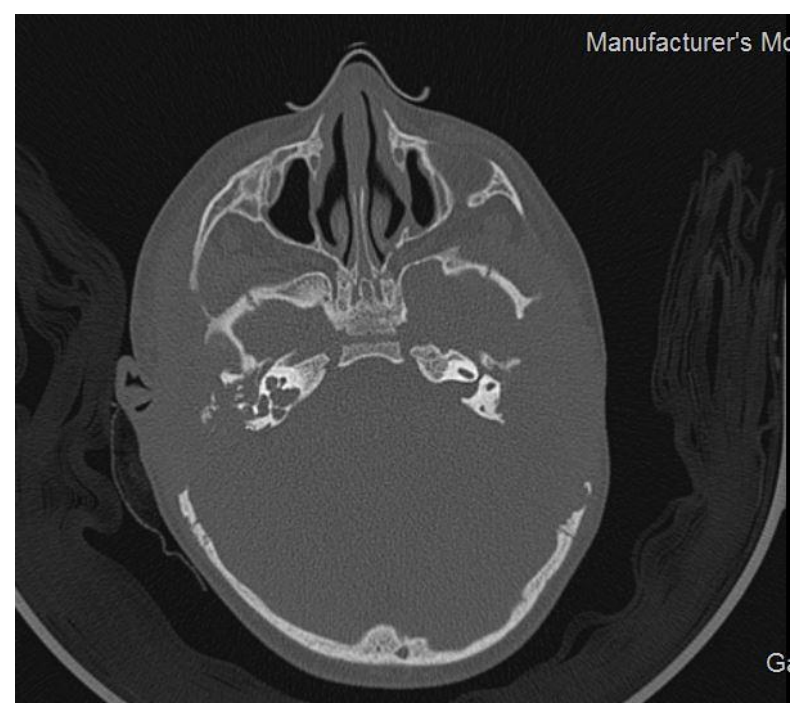

Fig. 2. CT scan showing bilateral temporal bone lysis.

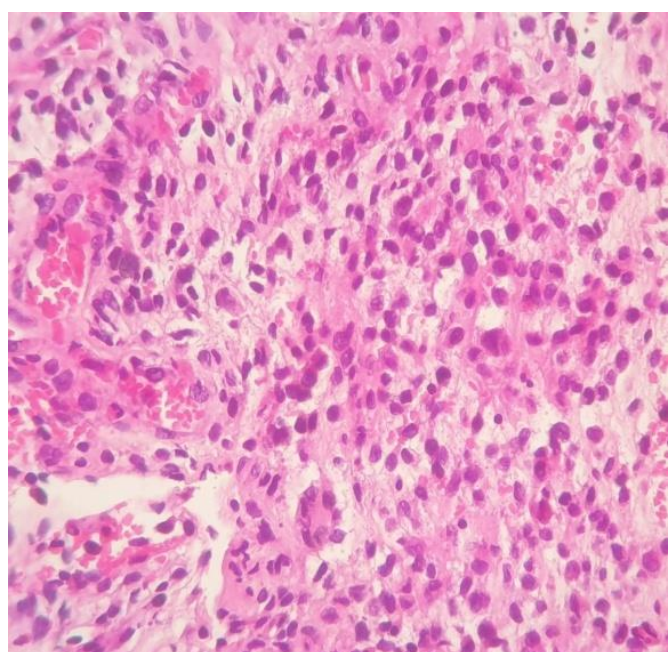

Fig. 3. Histological appearance of inflammatory and histiocytic cells.

\section{ClinicAl CASE 2}

A 2-year-old girl, with no particular medical history, presented with 3 months of chronic left otorrhea resistant to antibiotic treatment and progressing with no fever. Clinical examination objectified the presence of redness and induration in the left mastoid with the presence of an inflammatory polyp filling the entire left external auditory canal. Blood work was normal. CT scan revealed a left temporal bone lysis (Fig. 4). A biopsy and histological and immune-markers were performed and objectified an appearance similar to the first case, thus confirming the diagnosis of HL.

Both patients are treated with chemotherapy: DAL-XH-83 protocol

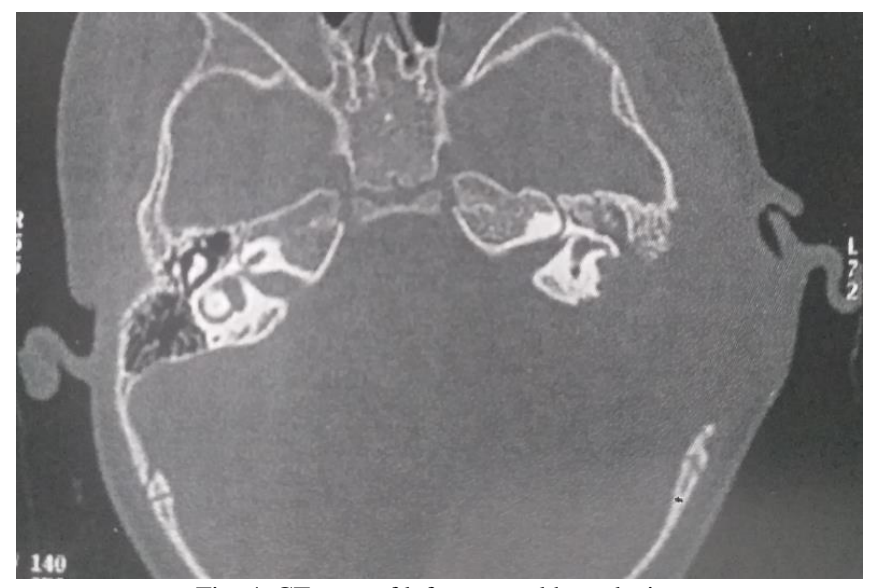

Fig. 4. CT scan of left temporal bone lysis.

\section{DISCUSSION}

HCL is a systemic disease that can affect several organs; preferably bone, skin, central nervous system, lungs, liver, and spleen. LCH can occur at any age. It mainly affects children between 1 and 4 years old. The incidence of the disease in children under 15 years of age is 2 to 9 cases per million per year, with a slight male predominance [2].

Clinical presentation is very variable and there are several clinical forms. HCL was formerly called Histiocytosis X and included three clinical forms, which are eosinophilic granuloma, Hand-Schüller Christian disease and LettererSiwe disease [3]. Currently, this nomenclature is abandoned; therefore, HCL is classified according to the number of lesions and organs affected. There is a distinction between mono-tissue monofocal or multifocal form and multi-tissue form [2]. Damage to certain organs such as lung, liver, spleen and bone marrow worsens the prognosis [1].

Otologic involvement in HCL varies from four to $61 \%$ depending on series [4]. It is secondary to temporal bone involvement, which is often unilateral but can be bilateral. Clinical presentation is similar to a chronic otitis media or otitis externa [5]. Radiologically, CT scan shows limited or diffuse bone lysis, preferentially affecting the mastoid, middle ear and outer ear.

Central diabetes insipidus is the most common endocrine manifestation; it is found in 15 to $50 \%$ of patients with $\mathrm{LCH}$ with involvement of the hypothalamic-pituitary axis [6]. It results from a defective secretion of the antidiuretic hormone (ADH). A deficiency in anterior pituitary hormones can also be found, and can be manifested by growth retardation, hypothyroidism and puberty delay.

We report the case of a 2-year-old child with HCL with bilateral temporal bone involvement associated with central pituitary involvement responsible for diabetes insipidus. Rare similar cases have been reported [7]-[9].

The diagnosis of HCL requires association of radioclinical presentation with a histological and immunomarkers appearance compatible with the disease. The identification of characteristic cells and the presence of specific markers, such as CD1a and/or CD207, allow a definitive diagnosis of the disease [10]. 


\section{CONCLUSION}

HCL is a rare condition that manifests in different clinical forms. The diagnosis is difficult and often delayed. Clinicians should become familiar with different manifestations suggesting the disease such as the presence of central diabetes insipidus or bilateral temporal diffuse bone lysis.

\section{CONFLICT OF INTEREST}

All authors declared that there is no conflict of interests.

\section{CONSENT}

Informed consent was obtained from the patient for publication of this case report and accompanying images.

\section{REFERENCES}

[1] Papadopoulou M, Panagopoulou P, Papadopoulou A, Hatzipantelis E, Efstratiou I, Galli-Tsinopoulou A, et al. The multiple faces of Langerhans cell histiocytosis in childhood: A gentle reminder. Molecular and clinical oncology. 2018;8(3):489-92.

[2] Leung AK, Lam JM, Leong KF. Childhood Langerhans cell histiocytosis: a disease with many faces. World Journal of Pediatrics. 2019:1-10.

[3] Modest MC, Garcia JJ, Arndt CS, Carlson ML. Langerhans cell histiocytosis of the temporal bone: a review of 29 cases at a single center. The Laryngoscope. 2016;126(8):1899-904.

[4] Majumder A, Wick CC, Collins R, Booth TN, Isaacson B, Kutz JW. Pediatric Langerhans cell histiocytosis of the lateral skull base. International journal of pediatric otorhinolaryngology. 2017;99:13540 .

[5] Zheng H, Xia Z, Cao W, Feng Y, Chen S, Li Y-H, et al. Pediatric Langerhans cell histiocytosis of the temporal bone: clinical and imaging studies of 27 cases. World journal of surgical oncology. 2018;16(1):72.

[6] Radojkovic D, Pesic M, Dimic D, Petkovic TR, Radenkovic S, VelojicGolubovic M, et al. Localised Langerhans cell histiocytosis of the hypothalamic-pituitary region: case report and literature review. Hormones. 2018;17(1):119-25.

[7] Allen A, Matrova E, Ozgen B, Redleaf M, Emmadi R, Saran N. Langerhans' cell histiocytosis of the temporal bone in an adult with central diabetes insipidus. Radiology case reports. 2019;14(7):847-50.

[8] Guo Y, Ning F, Wang G, Li X, Liu J, Yuan Y, et al. Retrospective study of Langerhans cell histiocytosis in ear, nose and neck. American Journal of Otolaryngology. 2019:102369.

[9] Modugno G, Brandolini C, Magnani G, Ferri G, Sabattini E, Pirodda A. Langerhans cell histiocytosis: bilateral temporal bone involvement in an adult with diabetes insipidus. B-ENT. 2010;6(1):67-72.

[10] Jezierska M, Stefanowicz J, Romanowicz G, Kosiak W, Lange M. Langerhans cell histiocytosis in children-a disease with many faces. Recent advances in pathogenesis, diagnostic examinations and treatment. Advances in Dermatology and Allergology/Postępy Dermatologii i Alergologii. 2018;35(1):6. 